\title{
LIFTING CONNECTIONS TO THE $r$-JET PROLONGATION OF THE COTANGENT BUNDLE
}

\author{
WŁODZIMIERZ M. MIKULSKI
}

\begin{abstract}
We show that the problem of finding all $\mathcal{M} f_{m}$-natural operators $C: Q \rightsquigarrow$ $Q J^{r} T^{*}$ lifting classical linear connections $\nabla$ on $m$-manifolds $M$ into classical linear connections $C_{M}(\nabla)$ on the $r$-jet prolongation $J^{r} T^{*} M$ of the cotangent bundle $T^{*} M$ of $M$ can be reduced to that of finding all $\mathcal{M} f_{m}$-natural operators $D: Q \rightsquigarrow \stackrel{p}{\otimes} T \otimes$ $\stackrel{q}{\otimes} T^{*}$ sending classical linear connections $\nabla$ on $M$ into tensor fields $D_{M}(\nabla)$ of type $(p, q)$ on $M$.
\end{abstract}

\section{INTRODUCTION}

All manifolds are assumed to be smooth, Hausdorff, finite dimensional, and without boundaries. Maps are assumed to be smooth (of class $C^{\infty}$ ). The category of $m$-dimensional manifolds and their embeddings is denoted by $\mathcal{M} f_{m}$.

In [5], M. Kureš described completely all $\mathcal{M} f_{m}$-natural operators $B: Q_{\tau} \rightsquigarrow$ $Q T^{*}$ lifting torsion free classical linear connections $\nabla$ on $m$-manifolds $M$ into classical linear connections $B_{M}(\nabla)$ on the cotangent bundle $T^{*} M$ of $M$.

In [4], the authors studied a similar problem of describing all $\mathcal{M} f_{m}$-natural operators $B: Q \rightsquigarrow Q\left(\bigotimes^{k} T^{*}\right)$ transforming classical linear connections $\nabla$ on $m$ manifolds $M$ into classical linear connections $B_{M}(\nabla)$ on the $k$-th tensor power $\bigotimes^{k} T^{*} M$ of the cotangent bundle $T^{*} M$ of $M$. They proved that this problem can be reduced to the well known one of describing all $\mathcal{M} f_{m}$-natural operators $D: Q \rightsquigarrow \stackrel{p}{\bigotimes} T \otimes \bigotimes^{q} T^{*}$ sending classical linear connections $\nabla$ on $m$-manifolds $M$ into tensor fields $D_{M}(\nabla)$ of type $(p, q)$ on $M$.

In [7], we investigated a similar problem of describing all $\mathcal{M} f_{m}$-natural operators $B: Q \rightsquigarrow Q T^{r *}$ lifting classical linear connections $\nabla$ on $m$-manifolds $M$ into classical linear connections $B_{M}(\nabla)$ on the $r$-th order cotangent bundle $T^{r *} M=J^{r}(M, \mathbb{R})_{0}$ of $M$. We proved that this problem can also be reduced to the well known one of describing all $D: Q \rightsquigarrow \stackrel{p}{\otimes} T \otimes \bigotimes^{q} T^{*}$.

In the present note, we consider a similar problem of describing all $\mathcal{M} f_{m}$-natural operators $C: Q \rightsquigarrow Q J^{r} T^{*}$ lifting classical linear connections $\nabla$ on $m$-manifolds $M$ into classical linear connections $C_{M}(\nabla)$ on the $r$-jet prolongation $J^{r} T^{*} M$ of the cotangent bundle $T^{*} M$ of $M$. Modifying paper [7], we show that this problem

$M S C$ (2010): primary 58A20; secondary 58A 32 .

Keywords: classical linear connection, natural operator. 
can be reduced to the well-known one of describing all

$D: Q \rightsquigarrow \stackrel{p}{\otimes} T \otimes \stackrel{q}{\otimes} T^{*}$, too.

We recall that the $r$-jet prolongation of the cotangent bundle is a functor $J^{r} T^{*}$ : $\mathcal{M} f_{m} \rightarrow \mathcal{V B}$ sending any $m$-manifold $M$ into $J^{r} T^{*} M$ (the vector bundle of $r$-jets of sections $M \rightarrow T^{*} M$ of the cotangent bundle $T^{*} M \rightarrow M$ of $M$ ) and any embedding $\varphi: M_{1} \rightarrow M_{2}$ of two $m$-manifolds into $J^{r} T^{*} \varphi: J^{r} T^{*} M_{1} \rightarrow J^{r} T^{*} M_{2}$ given by $J^{r} T^{*} \varphi\left(j_{x}^{r} \omega\right)=j_{\varphi(x)}^{r}\left(T^{*} \varphi \circ \omega \circ \varphi^{-1}\right), j_{x}^{r} \omega \in J^{r} T^{*} M$. If $r=0, J^{0} T^{*} M \stackrel{\sim}{=} T^{*} M$ (the usual cotangent bundle).

Further, we inform that a linear connection on a vector bundle $E$ over a manifold $M$ is a bilinear map $D: \mathcal{X}(M) \times \Gamma E \rightarrow \Gamma E$ such that $D_{f X} \sigma=f D_{X} \sigma$ and $D_{X} f \sigma=$ $X f \sigma+f D_{X} \sigma$ for any smooth map $f: M \rightarrow \mathbb{R}$, any vector field $X \in \mathcal{X}(M)$ on $M$ and any smooth section $\sigma \in \Gamma E$ of $E \rightarrow M$. In particular, a linear connection $\nabla$ in the tangent space $T M$ of $M$ is called a classical linear connection on $M$.

We also inform that a general definition of natural operators can be found in [3]. In particular, an $\mathcal{M} f_{m}$-natural operator $C: Q \rightsquigarrow Q J^{r} T^{*}$ is an $\mathcal{M} f_{m}$-invariant system $C=\left\{C_{M}\right\}_{M \in o b j\left(\mathcal{M} f_{m}\right)}$ of regular operators (functions)

$$
C_{M}: \underline{Q}(M) \rightarrow \underline{Q}\left(J^{r} T^{*} M\right)
$$

for any $m$-manifold $M$ where $\underline{Q}(M)$ is the set of all classical linear connections on $M$. More precisely, the $\mathcal{M} \overline{f_{m}}$-invariance of $C$ means that if $\nabla_{1} \in \underline{Q}\left(M_{1}\right)$ and $\nabla_{2} \in Q\left(M_{2}\right)$ are $\varphi$-related by an embedding $\varphi: M_{1} \rightarrow M_{2}$ of $m$-manifolds (i.e. $\varphi$ is $\left(\nabla_{1}, \overline{\nabla_{2}}\right)$-affine), then $C_{M_{1}}\left(\nabla_{1}\right)$ and $C_{M_{2}}\left(\nabla_{2}\right)$ are $J^{r} T^{*} \varphi$-related. The regularity means that $C_{M}$ transforms smoothly parametrized families of connections into smoothly parametrized ones.

Similarly, an $\mathcal{M} f_{m}$-natural operator (natural tensor) $D: Q \rightsquigarrow \stackrel{p}{\otimes} T \otimes \stackrel{q}{\otimes}^{*} T^{*}$ is an $\mathcal{M} f_{m}$-invariant system $D=\left\{D_{M}\right\}_{M \in o b j\left(\mathcal{M} f_{m}\right)}$ of regular operators

$$
D_{M}: \underline{Q}(M) \rightarrow \mathcal{T}^{p, q}(M)
$$

for any $M \in \mathcal{M} f_{m}$, where $\mathcal{T}^{p, q}(M)$ is the set of tensor fields of type $(p, q)$ on $M$.

By the general result in [6], since $J^{r} T^{*}: \mathcal{M} f \rightarrow \mathcal{V B}$ is a vector natural bundle, there exists an $\mathcal{M} f_{m}$-natural operator $C: Q \rightsquigarrow Q J^{r} T^{*}$. An explicit example of a natural operator $C: Q \rightsquigarrow Q J^{r} T^{*}$ (similar to Example 1 in [7]) will be presented in item 2 , too.

A full description of all $\mathcal{M} f_{m}$-natural operators $Q \rightsquigarrow \bigotimes^{p} T \otimes \bigotimes^{q} T^{*}$ transforming torsion free classical connections on $m$-manifolds into tensor fields of types $(p, q)$ can be found in Lemma in Section 33.4 in [3]. For the reader's convenience, we present this description. Each covariant derivative of the curvature $\mathcal{R}(\nabla) \in$ $C_{M}^{\infty}\left(\wedge^{2} T^{*} M \otimes T^{*} M \otimes T M\right)$ of a classical linear connection $\nabla$ is an $\left(\mathcal{M} f_{m^{-}}\right)$natural tensor. Further, every tensor multiplication of two natural tensors and every contraction on one covariant and one contravariant entry of a natural tensor gives a new natural tensor. Finally, we can multiply any natural tensor with a connection independent natural tensor, we can permute any number of entries in the tensor product and we can repeat these steps and take linear combinations. In this way, we can obtain any natural tensor of types $(p, q)$ depending on a torsion free classical linear connection. All natural tensors of a (not necessarily torsion free) classical 
linear connection $\nabla$ can be obtained provided we also include the torsion tensor $\mathcal{T}(\nabla)$ and their covariant derivatives in the above procedure.

\section{Preparations}

We are going to present an example of an $\mathcal{M} f_{m}$-natural operator $C^{(r)}: Q \rightsquigarrow$ $Q J^{r} T^{*}$. We start with the following important proposition (similar to Proposition 1 in [7]).

Proposition 2.1. Let $\nabla$ be a classical linear connection on $M$. Then, there is a (canonical in $\nabla)$ vector bundle isomorphism

$$
I_{\nabla}: J^{r} T^{*} M \rightarrow \bigoplus_{k=0}^{r} \bigodot^{k} T^{*} M \otimes T^{*} M
$$

covering the identity map of $M$.

Proof. We proceed as in the proof of Proposition 1 in [7]. Let $v \in T_{x}^{r *} M$, $x \in M$. Let $\varphi:(M, x) \rightarrow\left(\mathbb{R}^{m}, 0\right)$ be a $\nabla$-normal coordinate system with center $x$. We put

$$
I_{\nabla}(v)=I_{\nabla}^{\varphi}(v):=\bigoplus_{k=0}^{r} \bigodot^{k} T^{*} \varphi^{-1} \otimes T^{*} \varphi^{-1} \circ I \circ J^{r} T^{*} \varphi(v),
$$

where $I: J^{r} T_{0}^{*} \mathbb{R}^{m} \rightarrow \bigoplus_{k=1}^{r} \bigodot^{k} T_{0}^{*} \mathbb{R}^{m} \otimes T_{0}^{*} \mathbb{R}^{m}=\bigoplus_{k=0}^{r} \bigodot^{k} \mathbb{R}^{m *} \otimes \mathbb{R}^{m *}$ is the obvious $G L(m)$-invariant vector space isomorphism. If $\psi:(M, x) \rightarrow\left(\mathbb{R}^{m}, 0\right)$ is another $\nabla$-normal coordinate system with center $x$, then $\psi=A \circ \varphi$ (near $x$ ) for some $A \in G L(m)$. Using the $G L(m)$-invariance of $I$, we deduce that $I_{\nabla}^{\psi}(v)=$ $I_{\nabla}^{\varphi}(v)$. So, the definition of $I_{\nabla}(v)$ is independent of the choice of $\varphi$.

In [1], J. Gancarzewicz presented a canonical construction of a classical linear connection on the total space of a vector bundle $E$ over $M$ from a linear connection $D$ in $E$ by means of a classical linear connection $\nabla$ on $M$. For the reader's convenience, we present the construction. If $X$ is a vector field on $M$ and $\sigma$ is a section of $E$, then $D_{X} \sigma$ is a section of $E$. Further, let $X^{D}$ denote the horizontal lift of a vector field $X$ with respect to $D$. Moreover, using the translations in the individual fibres of $E$, we derive from every section $\sigma: M \rightarrow E$ a vertical vector field $\sigma^{V}$ on $E$ called the vertical lift of $\sigma$. In [1], J. Gancarzewicz proved the following fact.

Proposition 2.2. For every linear connection $D$ in a vector bundle $E$ over $M$ and every classical linear connection $\nabla$ on $M$, there exists a unique classical linear connection $\Theta=\Theta(D, \nabla)$ on the total space $E$ with the following properties

$$
\begin{gathered}
\Theta_{X^{D}} Y^{D}=\left(\nabla_{X} Y\right)^{D}, \Theta_{X^{D}} \sigma^{V}=\left(D_{X} \sigma\right)^{V}, \\
\Theta_{\sigma^{V}} X^{D}=0, \Theta_{\sigma^{V}} \sigma_{1}^{V}=0
\end{gathered}
$$

for all vector fields $X, Y$ on $M$ and all sections $\sigma, \sigma_{1}$ of $E$. 
It is well-known (see [2]) that every classical linear connection $\nabla$ on an $m$ manifold $M$ can be extended to a linear connection $D_{\nabla}^{(r)}=\nabla$ in $\bigoplus_{k=0}^{r} \bigodot^{k} T^{*} M \otimes$ $T^{*} M$ by

$$
\begin{aligned}
& \left(\nabla_{X} A\right)\left(X_{0}, \ldots, X_{k}\right)=X A\left(X_{0}, \ldots, X_{k}\right)-\sum_{i=0}^{k} A\left(X_{0}, \ldots, \nabla_{X} X_{i}, \ldots, X_{k}\right), \\
& A \in \Gamma\left(\bigodot^{k} T^{*} M \otimes T^{*} M\right), X_{0}, \ldots, X_{k} \in \mathcal{X}(M), k=0, \ldots, r .
\end{aligned}
$$

Now, we are in a position to present a natural operator $C^{(r)}: Q \rightsquigarrow Q J^{r} T^{*}$.

Example 2.3. As in Example 1 in [7], given a classical linear connection $\nabla$ on $M$, by Propositions 2.1 and 2.2 , we have the classical linear connection $\nabla^{(r)}$ on $J^{r} T^{*} M$ given by

$$
\nabla^{(r)}:=\left(I_{\nabla}\right)_{*}^{-1} \Theta\left(D_{\nabla}^{(r)}, \nabla\right)
$$

Clearly, the family $C^{(r)}: Q \rightsquigarrow Q J^{r} T^{*}$ of operators

$$
C_{M}^{(r)}: \underline{Q}(M) \rightarrow \underline{Q}\left(J^{r} T^{*} M\right), C_{M}^{(r)}(\nabla):=\nabla^{(r)},
$$

where $M \in \operatorname{obj}\left(\mathcal{M} f_{m}\right)$ and $\nabla \in \underline{Q}(M)$, is an $\mathcal{M} f_{m}$-natural operator.

\section{A Simple REDUCTion}

The set of all $\mathcal{M} f_{m}$-natural operators $C: Q \rightsquigarrow Q J^{r} T^{*}$ is an affine space with the corresponding vector space of all $\mathcal{M} f_{m}$-natural operators

$\Delta: Q \rightsquigarrow\left(\otimes^{2} T^{*} \otimes T\right) J^{r} T^{*}$ lifting classical linear connections $\nabla$ on $m$-manifolds $M$ into tensor fields $\Delta_{M}(\nabla)$ of type $(1,2)$ on $J^{r} T^{*} M$ (the definition is quite similar to that of natural operators $\left.Q \rightsquigarrow Q J^{r} T^{*}\right)$. Actually, given $\mathcal{M} f_{m}$-natural operators $C: Q \rightsquigarrow Q J^{r} T^{*}$ and $\Delta: Q \rightsquigarrow\left(\bigotimes^{2} T^{*} \otimes T\right) J^{r} T^{*}$ we have $\mathcal{M} f_{m}$-natural operator $C+\Delta: Q \rightsquigarrow Q J^{r} T^{*}$ given by

$$
(C+\Delta)_{M}(\nabla):=C_{M}(\nabla)+\Delta_{M}(\nabla), \nabla \in \underline{Q}(M), M \in \operatorname{obj}\left(\mathcal{M} f_{m}\right) .
$$

So, as in [7], to describe all $\mathcal{M} f_{m}$-natural operators $C: Q \rightsquigarrow Q J^{r} T^{*}$, it is sufficient to describe all $\mathcal{M} f_{m}$-natural operators $\Delta: Q \rightsquigarrow\left(\otimes^{2} T^{*} \otimes T\right) J^{r} T^{*}$. Further, because of Proposition 2.1, we can put $\bigoplus_{k=0}^{r} \bigodot^{k} T^{*} \otimes T^{*}$ instead of $J^{r} T^{*}$, and our problem of describing all $\mathcal{M} f_{m}$-natural operators $C: Q \rightsquigarrow Q J^{r} T^{*}$ is reduced to that of finding all $\mathcal{M} f_{m}$-natural operators

$$
\Delta: Q \rightsquigarrow\left(\bigotimes^{2} T^{*} \otimes T\right) \bigoplus_{k=0}^{r} \bigodot^{k} T^{*} \otimes T^{*}
$$

lifting classical linear connections $\nabla$ on $m$-manifolds into tensor fields $\Delta_{M}(\nabla)$ of type $(1,2)$ on $\bigoplus_{k=0}^{r} \bigodot^{k} T^{*} M \otimes T^{*} M$. 
As in [7], given a classical linear connection $\nabla$ on $M$ we have

$$
\begin{aligned}
& T_{v}\left(\bigoplus_{k=0}^{r} \bigodot \bigodot^{*} M \otimes T^{*} M\right)=V_{v}\left(\bigoplus_{k=0}^{r} \bigodot^{k} T^{*} M \otimes T^{*} M\right) \oplus H_{v}^{\nabla} \\
& \simeq \bigoplus_{k=0}^{r} \bigodot T_{x}^{*} M \otimes T_{x}^{*} M \oplus T_{x} M
\end{aligned}
$$

for any $v \in \bigoplus_{k=0}^{r} \bigodot^{k} T_{x}^{*} M \otimes T_{x}^{*} M, x \in M$ where $H_{v}^{\nabla}$ is the $\nabla$-horizontal subspace and the identification $\stackrel{\sim}{=}$ is the standard one. Then, by linear algebra,

$$
\begin{aligned}
& \left.T_{v}\left(\bigoplus_{k=0}^{r} \bigodot^{k} T^{*} M \otimes T^{*} M\right)\right)^{*} \otimes\left(T_{v}\left(\bigoplus_{k=0}^{r} \bigodot^{k} T^{*} M \otimes T^{*} M\right)\right)^{*} \\
\otimes & T_{v}\left(\bigoplus_{k=0}^{r} \bigodot^{k} T^{*} M \otimes T^{*} M\right) \\
= & \left(T_{x}^{*} M \otimes T_{x}^{*} M \otimes T_{x} M\right) \oplus \bigoplus_{l=0}^{r}\left(T_{x}^{*} M \otimes T_{x}^{*} M \otimes \bigodot^{l} T_{x}^{*} M \otimes T_{x}^{*} M\right) \\
& \bigoplus_{l=0}^{r}\left(T_{x}^{*} M \otimes \bigodot^{l} T_{x} M \otimes T_{x} M \otimes T_{x} M\right) \\
& \bigoplus_{l, l_{1}=0}^{r}\left(T_{x}^{*} M \otimes \bigodot T_{x} M \otimes T_{x} M \otimes \bigodot^{l} T_{x}^{*} M \otimes T_{x}^{*} M\right) \\
& \bigoplus_{l=0}^{r}\left(\bigodot^{l} T_{x} M \otimes T_{x} M \otimes T_{x}^{*} M \otimes T_{x} M\right) \\
& \bigoplus_{l, l_{1}=0}^{r}\left(\bigodot^{l} T_{x} M \otimes T_{x} M \otimes T_{x}^{*} M \otimes \bigodot^{l_{1}} T_{x}^{*} M \otimes T_{x}^{*} M\right) \\
\oplus & \bigoplus_{l, l_{1}=0}^{r}\left(\bigodot^{l} T_{x} M \otimes T_{x} M \otimes \bigodot^{l_{1}} T_{x} M \otimes T_{x} M \otimes T_{x} M\right) \\
\oplus & \bigoplus_{l, l_{1}, l_{2}=0}^{l}\left(\bigodot^{l} T_{x} M \otimes T_{x} M \otimes \bigodot^{l_{1}} T_{x} M \otimes T_{x} M \otimes \bigodot^{l_{2}} T_{x}^{*} M \otimes T_{x}^{*} M\right) .
\end{aligned}
$$

Consequently, our problem of finding of all $\mathcal{M} f_{m}$-natural operators $C: Q \rightsquigarrow$ $Q J^{r} T^{*}$ is reduced to that of finding systems $\Delta^{C}=\left(\left(\Delta^{1}\right), \ldots,\left(\Delta_{l, l_{1}, l_{2}}^{8}\right)\right)$ of systems $\left(\Delta^{1}\right), \ldots,\left(\Delta_{l, l_{1}, l_{2}}^{8}\right)$ of $\mathcal{M} f_{m}$-natural operators

$$
\begin{aligned}
& \Delta^{1}: Q \rightsquigarrow\left(\bigoplus_{k=0}^{r} \bigodot^{k} T^{*} \otimes T^{*}, T^{*} \otimes T^{*} \otimes T\right), \\
& \Delta_{l}^{2}: Q \rightsquigarrow\left(\bigoplus_{k=0}^{r} \bigodot^{k} T^{*} \otimes T^{*}, T^{*} \otimes T^{*} \otimes \bigodot T^{*} \otimes T^{*}\right),
\end{aligned}
$$




$$
\begin{aligned}
& \Delta_{l}^{3}: Q \rightsquigarrow\left(\bigoplus_{k=0}^{r} \bigodot^{k} T^{*} \otimes T^{*}, T^{*} \otimes \bigodot^{l} T \otimes T \otimes T\right), \\
& \Delta_{l, l_{1}}^{4}: Q \rightsquigarrow\left(\bigoplus_{k=0}^{r} \bigodot^{k} T^{*} \otimes T^{*}, T^{*} \otimes \bigodot^{l} T \otimes T \otimes \bigodot^{l_{1}} T^{*} \otimes T^{*}\right), \\
& \Delta_{l}^{5}: Q \rightsquigarrow\left(\bigoplus_{k=0}^{r} \bigodot^{k} T^{*} \otimes T^{*}, \bigodot T \otimes T \otimes T^{*} \otimes T\right), \\
& \Delta_{l, l_{1}}^{6}: Q \rightsquigarrow\left(\bigoplus_{k=0}^{r} \bigodot^{k} T^{*} \otimes T^{*}, \bigodot T \otimes T \otimes T^{*} \otimes \bigodot^{l_{1}} T^{*} \otimes T^{*}\right), \\
& \Delta_{l, l_{1}}^{7}: Q \rightsquigarrow\left(\bigoplus_{k=0}^{r} \bigodot^{k} T^{*} \otimes T^{*}, \bigodot^{l} T \otimes T \otimes \bigodot \bigodot^{l_{1}} T \otimes T \otimes T\right), \\
& \Delta_{l, l_{1}, l_{2}}^{8}: Q \rightsquigarrow\left(\bigoplus_{k=0}^{r} \bigodot^{k} T^{*} \otimes T^{*}, \bigodot T \otimes T \otimes \bigodot^{l} T \otimes T \otimes \bigodot^{l_{1}} T^{*} \otimes T^{*}\right)
\end{aligned}
$$

transforming classical linear connections $\nabla$ on $m$-manifolds $M$ into fibred maps $\Delta_{M}^{1}(\nabla): \bigoplus_{k=0}^{r} \bigodot^{k} T^{*} M \otimes T^{*} M \rightarrow T^{*} M \otimes T^{*} M \otimes T M, \ldots, \Delta_{l, l_{1}, l_{2}}^{8} M(\nabla):$ $\bigoplus_{k=0}^{r} \bigodot^{k} T^{*} M \otimes T^{*} M \rightarrow \bigodot^{l} T M \otimes T M \otimes \bigodot^{l_{1}} T M \otimes T M \otimes \bigodot^{l_{2}} T^{*} M \otimes T^{*} M$ covering the identity map of $M$, where $l, l_{1}, l_{2}=0, \ldots, r$.

\section{A MORE REDUCTION}

To obtain a more reduction than the above one, we need a preparation.

As in [7], a tensor natural sub-bundle (of type $(p, q)$ ) is a natural vector bundle $F: \mathcal{M} f_{m} \rightarrow \mathcal{V B}$ such that (modulo a natural vector bundle isomorphism) $F M \subset$ $\bigotimes^{p} T M \otimes \bigotimes^{q} T^{*} M$ and $F \varphi=\bigotimes^{p} T \varphi \otimes \bigotimes^{q} T^{*} \varphi \mid F M$ for any $m$-manifold $M$ and any $\mathcal{M} f_{m}$-map $\varphi: M \rightarrow M^{1}$.

Proposition 4.1. Let $F: \mathcal{M} f_{m} \rightarrow \mathcal{V B}$ be a tensor natural sub-bundle of type $(p, q)$. The $\mathcal{M} f_{m}$-natural operators $B: Q \rightsquigarrow\left(\bigoplus_{k=0}^{r} \bigodot^{k} T^{*} \otimes T^{*}, F\right)$ transforming classical linear connections $\nabla$ on m-manifolds $M$ into fibred maps $B_{M}(\nabla)$ : $\bigoplus_{k=0}^{r} \bigodot^{k} T^{*} M \otimes T^{*} M \rightarrow F M$ covering $i d_{M}$ are in bijection with the systems $E=\left(E^{\left(k_{1}, \ldots, k_{j}\right)}\right)$ of $\mathcal{M} f_{m}$-natural operators

$E^{\left(k_{1}, \ldots, k_{j}\right)}: Q \rightsquigarrow\left(\left(\bigodot^{k_{1}} T \otimes T\right) \odot \ldots \odot\left(\bigodot^{k_{j}} T \otimes T\right)\right) \otimes F$ for systems $\left(k_{1}, \ldots, k_{j}\right)$ of integers $k_{1}, \ldots, k_{j}$ with $0 \leq k_{1} \leq \ldots \leq k_{j} \leq r, k_{1}+\ldots+k_{j} \leq q-p-j$, $j=0,1,2, \ldots$ If $j=0,\left(k_{1}, \ldots, k_{j}\right)=\emptyset$, and $E^{\emptyset}: Q \rightsquigarrow F$. If $q-p-j<0$, any $B$ is the zero operator. (For $\odot$, see below.)

More precisely, the natural operator $B^{E}: Q \rightsquigarrow\left(\bigoplus_{k=0}^{r} \bigodot^{k} T^{*} \otimes T^{*}, F\right)$ corresponding to a system $E=\left(E^{\left(k_{1}, \ldots, k_{j}\right)}\right)$ (as above) is defined by

$$
B_{M}^{E}(\nabla)_{x}(v)=\sum<E_{M}^{\left(k_{1}, \ldots, k_{j}\right)}(\nabla)_{x}, v_{k_{1}} \otimes \ldots \otimes v_{k_{j}}>,
$$


$\nabla \in Q(M), M \in \operatorname{obj}\left(\mathcal{M} f_{m}\right), x \in M, v=\left(v_{0}, \ldots, v_{r}\right) \in \bigoplus_{k=0}^{r} \bigodot^{k} T_{x}^{*} M \otimes T_{x}^{*} M$, where the (finite) sum $\sum$ is over all systems $\left(k_{1}, \ldots, k_{j}\right)$ of integers with $0 \leq k_{1} \leq$ $\ldots \leq k_{j} \leq r, k_{1}+\ldots+k_{j} \leq q-p-j, j=0,1,2, \ldots$

Conversely, the system $E^{B}=\left(E^{B ;\left(k_{1}, \ldots, k_{j}\right)}\right)$ corresponding to a natural operator $B \rightsquigarrow\left(\bigoplus_{k=0}^{r} \bigodot^{k} T^{*} \otimes T^{*}, F\right)$ is well-defined by

$$
\begin{aligned}
& <E_{M}^{B ;\left(k_{1}, \ldots, k_{j}\right)}(\nabla)_{x}, v_{k_{1}} \otimes \ldots \otimes v_{k_{j}}> \\
& =\frac{1}{\alpha !} \frac{\partial}{\partial t^{k_{1}}} \cdots \frac{\partial}{\partial t^{k_{j}}} B_{M}(\nabla)_{x}\left(t^{0} v_{0}, \ldots, t^{r} v_{r}\right)_{\left.\right|_{t^{0}, \ldots, t^{r}=0}}
\end{aligned}
$$

where $v=\left(v_{0}, \ldots, v_{r}\right)=\bigoplus_{k=0}^{r} \bigodot^{k} T_{x}^{*} M \otimes T_{x}^{*} M, x \in M, \alpha=1_{k_{1}}+\ldots+1_{k_{j}} \in \mathbb{N}^{r}$.

As in [7], in Proposition 4.1, we used the following notation. Given a sequence $V_{0}, \ldots, V_{r}$ of different vector spaces and a system $\left(k_{1}, \ldots, k_{j}\right)$ of integers with $0 \leq$ $k_{1} \leq \ldots \leq k_{j} \leq r, V_{k_{1}} \odot \ldots \odot V_{k_{j}}$ denotes the factor space $V_{k_{1}} \otimes \ldots \otimes V_{k_{j}} / \sim$ where, for any $u, w \in V_{k_{1}} \otimes \ldots \otimes V_{k_{j}}, u \sim w$ iff $<u, \varphi_{k_{1}} \otimes \ldots \otimes \varphi_{k_{j}}>=<w, \varphi_{k_{1}} \otimes \ldots \varphi_{k_{j}}>$ (the usual pairing (contraction)) for any $\left(\varphi_{0}, \ldots, \varphi_{r}\right) \in \bigoplus_{k=0}^{r} V_{k}^{*}$.

Proof. The proof of Proposition 4.1 is almost the same as that of Proposition 3 in [7]. By the non-linear Petree theorem (see [3]) $B$ is of finite order. Further, by the invariance with respect to manifold charts, $B$ is determined by the values

$$
\left(B_{\mathbb{R}^{m}}(\nabla)\right)_{0}(v) \in F_{0} \mathbb{R}^{m}
$$

for all classical linear connections $\nabla$ on $\mathbb{R}^{m}$ and all points $v=\left(v_{0}, \ldots, v_{r}\right) \in$ $\bigoplus_{k=0}^{r} \bigodot^{k} T_{0}^{*} \mathbb{R}^{m} \otimes T_{0}^{*} \mathbb{R}^{m}$. We can assume that the coordinates (symbols) of $\nabla$ are polynomials of a degree that is the finite order of $B$. Next, by the invariance of $B$ with respect to the homotheties, we have

$$
B_{\mathbb{R}^{m}}\left(\left(t i d_{\mathbb{R}^{m}}\right)_{*} \nabla\right)_{0}\left(\bigoplus_{k=0}^{r} \bigodot^{k} T^{*}\left(t i d_{\mathbb{R}^{m}}\right) \otimes T^{*}\left(t i d_{\mathbb{R}^{m}}\right)(v)\right)=t^{p-q} B_{\mathbb{R}^{m}}(\nabla)_{0}(v)
$$

for $t>0$. So, the homogeneous function theorem and the Taylor theorem end the proof.

\section{Corollaries}

Applying Proposition 4.1 to natural operators $\Delta^{1}, \ldots, \Delta_{l, l_{1}, l_{2}}^{8}$ in item 3 , we obtain.

Corollary 5.1. For $l=0, \ldots, r$ any $\mathcal{M} f_{m}$-natural operator $\Delta_{l}^{3}: Q \rightsquigarrow$ $\left(\bigoplus_{k=0}^{r} \bigodot^{k} T^{*} M \otimes T^{*}, T^{*} \oplus \bigodot^{l} T \otimes T \otimes T\right)$ is the zero one.

Corollary 5.2. For $l=0, \ldots, r$ any $\mathcal{M} f_{m}$-natural operator $\Delta_{l}^{5}: Q \rightsquigarrow$ $\left(\bigoplus_{k=0}^{r} \bigodot^{k} T^{*} \otimes T^{*}, \bigodot^{l} T \otimes T \otimes T^{*} \otimes T\right)$ is the zero one.

Corollary 5.3. For $l, l_{1}=0, \ldots, r$ any $\mathcal{M} f_{m}$-natural operator $\Delta_{l, l_{1}}^{7}: Q \rightsquigarrow$ $\left(\bigoplus_{k=0}^{r} \bigodot^{k} T^{*} \otimes T^{*}, \bigodot^{l} T \otimes T \otimes \bigodot^{l_{1}} T \otimes T \otimes T\right)$ is the zero one. 
Corollary 5.4. The $\mathcal{M} f_{m}$-natural operators $\Delta^{1}: Q \rightsquigarrow\left(\bigoplus_{k=0}^{r} \bigodot^{k} T^{*} \otimes T^{*}, T^{*} \otimes T^{*} \otimes T\right)$ are in (the) bijection with the systems $E^{\Delta^{1}}=\left(E^{\Delta^{1} ; \emptyset}, E^{\Delta^{1} ;(0)}\right)$ of $\mathcal{M} f_{m}$-natural operators $E^{\Delta^{1} ; \emptyset}: Q \rightsquigarrow T^{*} \otimes T^{*} \otimes T$ and $E^{\Delta^{1} ;(0)}: Q \rightsquigarrow T \otimes T^{*} \otimes T^{*} \otimes T$.

Corollary 5.5. Given natural numbers $l=0, \ldots, r$ the $\mathcal{M} f_{m}$-natural operators $\Delta_{l}^{2}: Q \rightsquigarrow\left(\bigoplus_{k=0}^{r} \bigodot^{k} T^{*} \otimes T^{*}, T^{*} \otimes T^{*} \otimes \bigodot^{l} T^{*} \otimes T^{*}\right)$ are in (the) bijection with the systems $E^{\Delta_{l}^{2}}=\left(E^{\Delta_{l}^{2} ;\left(k_{1}, \ldots, k_{j}\right)}\right)$ of $\mathcal{M} f_{m}$-natural operators $E^{\Delta_{l}^{2} ;\left(k_{1}, \ldots, k_{j}\right)}: Q \rightsquigarrow$ $\left(\left(\bigodot_{1}^{k_{1}} T \otimes T\right) \odot \ldots \odot\left(\bigodot^{k_{j}} T \otimes T\right)\right) \otimes T^{*} \otimes T^{*} \otimes \bigodot^{l} T^{*} \otimes T^{*}$ for systems $\left(k_{1}, \ldots, k_{j}\right)$ of integers with $0 \leq k_{1} \leq \ldots \leq k_{j} \leq r, k_{1}+\ldots+k_{j} \leq l+3-j, j=0,1,2, \ldots$.

Corollary 5.6. Given natural numbers $l, l_{1}=0, \ldots, r$ the $\mathcal{M} f_{m}$-natural operators $\Delta_{l, l_{1}}^{4}: Q \rightsquigarrow\left(\bigoplus_{k=0}^{r} \bigodot^{k} T^{*} \otimes T^{*}, T^{*} \otimes \bigodot^{l} T \otimes T \otimes \bigodot^{l_{1}} T^{*} \otimes T^{*}\right)$ are in (the) bijection with the systems $E^{\Delta_{l, l_{1}}^{4}}=\left(E^{\Delta_{l, l_{1}}^{4} ;\left(k_{1}, \ldots, k_{j}\right)}\right)$ of $\mathcal{M} f_{m}$-natural operators $E^{\Delta_{l, l_{1}}^{4} ;\left(k_{1}, \ldots, k_{j}\right)}: Q \rightsquigarrow\left(\left(\bigodot^{k_{1}} T \otimes T\right) \odot \ldots \odot\left(\bigodot^{k_{j}} T \otimes T\right)\right) \otimes T^{*} \otimes \stackrel{l}{\odot} T \otimes T \otimes \bigodot^{l_{1}} T^{*} \otimes T^{*}$ for systems $\left(k_{1}, \ldots, k_{j}\right)$ of integers with $0 \leq k_{1} \leq \ldots \leq k_{j} \leq r, k_{1}+\ldots+k_{j} \leq$ $l_{1}+1-l-j, j=0,1, \ldots$

Corollary 5.7. Given natural numbers $l, l_{1}=0, \ldots, r$ the $\mathcal{M} f_{m}$-natural operators $\Delta_{l, l_{1}}^{6}: Q \rightsquigarrow\left(\bigoplus_{k=0}^{r} \bigodot^{k} T^{*} \otimes T^{*}, \bigodot^{l} T \otimes T \otimes T^{*} \otimes \bigodot^{l_{1}} T^{*} \otimes T^{*}\right)$ are in (the) bijection with the systems $E^{\Delta_{l, l_{1}}^{6}}=\left(E^{\Delta_{l, l_{1}}^{6} ;\left(k_{1}, \ldots, k_{j}\right)}\right)$ of $\mathcal{M} f_{m}$-natural operators $E^{\Delta_{l, l_{1}}^{6} ;\left(k_{1}, \ldots, k_{j}\right)}: Q \rightsquigarrow\left(\left(\bigodot^{k_{1}} T \otimes T\right) \odot \ldots \odot\left(\bigodot^{k_{j}} T \otimes T\right)\right) \otimes \bigodot^{l} T \otimes T \otimes T^{*} \otimes \bigodot^{l_{1}} T^{*} \otimes T^{*}$ for systems $\left(k_{1}, \ldots, k_{j}\right)$ of integers with $0 \leq k_{1} \leq \ldots \leq k_{j} \leq r, k_{1}+\ldots+k_{j} \leq$ $l_{1}+1-l-j, j=0,1, \ldots$

Corollary 5.8. Given natural numbers $l, l_{1}, l_{2}=0, \ldots, r$ the $\mathcal{M} f_{m}$-natural operators $\Delta_{l, l_{1}, l_{2}}^{8}: Q \rightsquigarrow\left(\bigoplus_{k=0}^{r} \bigodot^{k} T^{*} \otimes T^{*}, \bigodot^{l} T \otimes T \otimes \bigodot^{l_{1}} T \otimes T \otimes \bigodot^{l_{2}} T^{*} \otimes T^{*}\right)$ are in (the) bijection with the systems $E^{\Delta_{l, l_{1}, l_{2}}^{8}}=\left(E^{\Delta_{l, l_{1}, l_{2}}^{8} ;\left(k_{1}, \ldots, k_{j}\right)}\right)$ of $\mathcal{M} f_{m}$-natural operators

$E^{\Delta_{l, l_{1}, l_{2}}^{8} ;\left(k_{1}, \ldots, k_{j}\right)}: Q \rightsquigarrow\left(\left(\bigodot^{k_{1}} T \otimes T\right) \odot \ldots \odot\left(\bigodot^{k_{j}} T \otimes T\right)\right) \otimes \bigodot^{l} T \otimes T \otimes \bigodot^{l_{1}} T \otimes$ $T \otimes \bigodot^{l_{2}} T^{*} \otimes T^{*}$ for systems $\left(k_{1}, \ldots, k_{j}\right)$ of integers with $0 \leq k_{1} \leq \ldots \leq k_{j} \leq r$, $k_{1}+\ldots+k_{j} \leq l_{2}-l_{1}-l-1-j, j=0,1, \ldots$

\section{The MAIN Result}

Summing up, we have proved the following result.

Theorem 6.1. The $\mathcal{M} f_{m}$-natural operators $C: Q \rightsquigarrow Q T^{r *}$ are in (the) bijection with the systems $\Delta^{C}=\left(\left(\Delta^{1}\right),\left(\Delta_{l}^{2}\right),\left(\Delta_{l, l_{1}}^{4}\right),\left(\Delta_{l, l_{1}}^{6}\right),\left(\Delta_{l, l_{1}, l_{2}}^{8}\right)\right)$ of systems $\left(\Delta^{1}\right), \ldots,\left(\Delta_{i, l_{1}, l_{2}}^{8}\right)$ of $\mathcal{M} f_{m}$-natural operators corresponding to systems of $\mathcal{M} f_{m^{-}}$ natural operators (of the form $Q \rightsquigarrow \bigotimes^{p} T \otimes \bigotimes^{q} T^{*}$ ) as in Corollaries 5.4-5.8. 
If $r=0$, then $l, l_{1}, l_{2}=0$ only. Consequently $E^{\Delta^{1}}=\left(E^{\Delta^{1} ; \emptyset}, E^{\Delta^{1} ;(0)}\right), E^{\Delta_{0}^{2}}=$ $\left(E^{\Delta_{0}^{2} ; \emptyset}, E^{\Delta_{0}^{2} ;(0)}, E^{\Delta_{0}^{2} ;(0,0)}, E^{\Delta_{0}^{2} ;(0,0,0)}\right), E^{\Delta_{0,0}^{4}}=\left(E^{\Delta_{0,0}^{4} ; \emptyset}, E^{\Delta_{0,0}^{4},(0)}\right), E^{\Delta_{0,0,0}^{8}}=(0)$, $E^{\Delta_{0,0}^{6}}=\left(E^{\Delta_{0,0}^{6} ; \emptyset}, E^{\Delta_{0,0}^{6} ;(0)}\right)$. So, Theorem 6.1 for $r=0$ can be read as follows.

The $\mathcal{M} f_{m}$-natural operators $C: Q \rightsquigarrow Q T^{*}$ lifting classical linear connections to the cotangent bundle are in the bijection with the 10 -tuples $\left(E^{\Delta^{1} ; \emptyset}, \ldots, E^{\Delta_{0,0}^{6} ;(0)}\right)$ containing of $\mathcal{M} f_{m}$-natural operators

$$
\begin{aligned}
& E^{\Delta^{1} ; \emptyset}: Q \rightsquigarrow T^{*} \otimes T^{*} \otimes T, \\
& E^{\Delta^{1} ;(0)}: Q \rightsquigarrow T \otimes T^{*} \otimes T^{*} \otimes T, \\
& E^{\Delta_{0}^{2} ; \emptyset}: Q \rightsquigarrow T^{*} \otimes T^{*} \otimes T^{*}, \\
& E^{\Delta_{0}^{2} ;(0)}: Q \rightsquigarrow T \otimes T^{*} \otimes T^{*} \otimes T^{*}, \\
& E^{\Delta_{0}^{2} ;(0,0)}: Q \rightsquigarrow(T \odot T) \otimes T^{*} \otimes T^{*} \otimes T^{*}, \\
& E^{\Delta_{0}^{2} ;(0,0,0)}: Q \rightsquigarrow(T \odot T \odot T) \otimes T^{*} \otimes T^{*} \otimes T^{*}, \\
& E^{\Delta_{0,0}^{4} ; \emptyset}: Q \rightsquigarrow T^{*} \otimes T \otimes T^{*}, \\
& E^{\Delta_{0,0}^{4} ;(0)}: Q \rightsquigarrow T \otimes T^{*} \otimes T \otimes T^{*}, \\
& E^{\Delta_{0,0}^{6} ; \emptyset}: Q \rightsquigarrow T^{*} \otimes T \otimes T^{*}, \\
& E^{\Delta_{0,0}^{6} ;(0)}: Q \rightsquigarrow T \otimes T^{*} \otimes T \otimes T^{*} .
\end{aligned}
$$

Using the description of $\mathcal{M} f_{m}$-natural operators $Q \rightsquigarrow \bigotimes^{p} T \otimes \bigotimes^{q} T^{*}$ of [3] (see item 1), we can explicitly describe the above 10-tuples. For example, any $\mathcal{M} f_{m}$-natural operator $E^{\Delta^{1} ; \emptyset}: Q \rightsquigarrow T^{*} \otimes T^{*} \otimes T$ is the linear combination (with real coefficients) of three $\mathcal{M} f_{m}$-natural operators (the connection torsion operator $T_{\nabla}$, the operator $\delta_{M} \otimes C_{1}^{1} T_{\nabla}$ (the tensor multiplication of the identity tensor field $\delta_{M}: T M \rightarrow T M$ and the contraction of the connection torsion) and the operator $\left.C_{1}^{1} T_{\nabla} \otimes \delta_{M}^{*}\right)$. In the case of torsion free connections, any such operator is the zero one. Similarly, any $\mathcal{M} f_{m}$-natural operator $E^{\Delta^{1} ;(0)}: Q \rightsquigarrow T \otimes T^{*} \otimes T^{*} \otimes T$ is the linear combination of two connection independent natural tensors (from the identity tensor $T M \otimes T M \rightarrow T M \otimes T M$ by means of the permutations of indices), e.t.c. In this way, we may reobtain (in another form) the result of M. Kureš [5] (in the case of natural operators $\left.Q_{\tau} \rightsquigarrow Q T^{*}\right)$ and get a more general similar result in the case of not necessarily torsion free connections.

The explanation of our result in the case $r=1$ (i.e. in the case of natural operators $Q \rightsquigarrow Q J^{1} T^{*}$ lifting classical linear connections to the first jet prolongation of the cotangent bundle) is more sophisticated but feasible. Indeed, if $r=1$, then $l, l_{1}, l_{2}=0,1$. Consequently $E^{\Delta^{1}}=\left(E^{\Delta^{1} ; \emptyset}, E^{\Delta^{1} ;(0)}\right)$. Next, $E^{\Delta_{0}^{2}}=$ $\left(E^{\Delta_{0}^{2} \emptyset}, E^{\Delta_{0}^{2} ;(0)}, E^{\Delta_{0}^{2} ;(1)}, E^{\Delta_{0}^{2} ;(0,0)}, E^{\Delta_{0}^{2} ;(0,1)}, E^{\Delta_{0}^{2} ;(1,1)}, E^{\Delta_{0}^{2} ;(0,0,0)}, E^{\Delta_{0}^{2} ;(0,0,1)}\right.$, $\left.E^{\Delta_{0}^{2} ;(0,1,1)}, E^{\Delta_{0}^{2} ;(1,1,1)}\right)$ and $E^{\Delta_{1}^{2}}=\left(E^{\Delta_{1}^{2} ; \emptyset}, E^{\Delta_{1}^{2} ;(0)}, E^{\Delta_{1}^{2} ;(1)}, E^{\Delta_{1}^{2} ;(0,0)}, E^{\Delta_{1}^{2} ;(0,1)}\right.$, $E^{\Delta_{1}^{2} ;(1,1)}, E^{\Delta_{1}^{2} ;(0,0,0)}, E^{\Delta_{1}^{2} ;(0,0,1)}, E^{\Delta_{1}^{2} ;(0,1,1)}, E^{\Delta_{1}^{2} ;(1,1,1)}, E^{\Delta_{1}^{2} ;(0,0,0,0)}, E^{\Delta_{1}^{2} ;(0,0,0,1)}$, $\left.E^{\Delta_{1}^{2} ;(0,0,1,1)}, E^{\Delta_{1}^{2} ;(0,1,1,1)}, E^{\Delta_{1}^{2} ;(1,1,1,1)}\right)$. Next $E^{\Delta_{1,0}^{4}}=\left(E^{\Delta_{1,0}^{4} ; \emptyset} ; E^{\Delta_{1,0}^{4} ;(0)}, E^{\Delta_{1,0}^{4} ;(1)}\right.$, $\left.E^{\Delta_{1,0}^{4} ;(0,0)}, E^{\Delta_{1,0}^{4} ;(0,1)}, E^{\Delta_{1,0}^{4} ;(1,1)}\right), E^{\Delta_{0,0}^{4}}=\left(E^{\Delta_{0,0}^{4} ; \emptyset}, E^{\Delta_{0,0}^{4} ;(0)}, E^{\Delta_{0,0}^{4} ;(1)}\right), E^{\Delta_{1,1}^{4}}=$ $\left(E^{\Delta_{1,1}^{4} ; \emptyset}, E^{\Delta_{1,1}^{4} ;(0)}, E^{\Delta_{1,1}^{4} ;(1)}\right)$ and $E^{\Delta_{0,1}^{4}}=\left(E^{\Delta_{0,1}^{4} ; \emptyset}\right)$. Similarly $E^{\Delta_{0,1}^{6}}=\left(E^{\Delta_{0,1}^{6} ; \emptyset}\right.$, 
$\left.E^{\Delta_{0,1}^{6} ;(0)}, E^{\Delta_{0,1}^{6} ;(1)}, E^{\Delta_{0,1}^{6} ;(0,0)}, E^{\Delta_{0,1}^{6} ;(0,1)}, E^{\Delta_{0,1}^{6} ;(1,1)}\right), E^{\Delta_{0,0}^{6}}=\left(E^{\Delta_{0,0}^{6} ; \emptyset}, E^{\Delta_{0,0}^{6} ;(0)}\right.$, $\left.E^{\Delta_{0,0}^{6} ;(1)}\right), E^{\Delta_{1,1}^{6}}=\left(E^{\Delta_{1,1}^{6} ; \emptyset}, E^{\Delta_{1,1}^{6} ;(0)}, E^{\Delta_{1,1}^{6} ;(1)}\right)$ and $E^{\Delta_{1,0}^{6}}=\left(E^{\Delta_{1,0}^{6} ; \emptyset}\right)$. Next $E^{\Delta_{0,0,1}^{8}}=\left(E^{\Delta_{0,0,1}^{8} ; \emptyset}\right)$, and $E^{\Delta_{l, l_{1}, l_{2}}^{8}}=(0)$ if $l=1$ or $l_{1}=1$ or $l_{2}=0$.

So, Theorem 6.1 for $r=1$ can be read as follows.

The $\mathcal{M} f_{m}$-natural operators $Q \rightsquigarrow Q J^{1} T^{*}$ are in (the) bijection with the 54tuples of $\mathcal{M} f_{m}$-natural operators

$$
\begin{aligned}
& E^{\Delta^{1} ; \emptyset}: Q \rightsquigarrow T^{*} \otimes T^{*} \otimes T, \\
& E^{\Delta_{0,0,1}^{8} ; \emptyset}: Q \rightsquigarrow T \otimes T \otimes T^{*} \otimes T^{*} .
\end{aligned}
$$

Clearly, the dots denote the respective types $\mathcal{M} f_{m}$-natural operators (we do not present the dots explicitly because this would require about two pages). Using the description of $\mathcal{M} f_{m}$-natural operators $Q \rightsquigarrow \bigotimes^{p} T \otimes \bigotimes^{q} T^{*}$ of [3] (see item 1) we may describe explicitly all above 54-tuples and describe explicitly all $\mathcal{M} f_{m}$-natural operators $C: Q \rightsquigarrow Q J^{1} T^{*}$.

\section{REFERENCES}

[1] J. Gancarzewicz, Horizontal lift of connections to a natural vector bundle, Differential geometry, Proc. 5th Int. Colloq., Santiago de Compostela, Spain 1984, Res. Notes in Math. 131, Pitman, 1985, 318-341.

[2] S. Kobayashi and K. Nomizu, Foundations of Differential Geometry, Vol. I, J. WileyInterscience, 1963.

[3] I. Kolář, P. W. Michor and J. Slovák, Natural Operations in Defferential Geometry, SpringerVerlag, Berlin, 1993.

[4] J. Kurek and W. M. Mikulski, The natural operators lifting connections to tensor powers of the cotangent bundle, Miskolc Math. Notes 14 (2013), 517-524.

[5] M. Kureš, Natural lifts of classical linear connections to the cotangent bundle, Suppl. Rend. Circ. Mat. Palermo, II. Ser. 43 (1996), 181-187.

[6] W. M. Mikulski, The natural bundles admitting natural lifting of linear connections, Demonstr. Math. 39 (2006), 223-132.

[7] W. M. Mikulski, The natural operators lifting connections to higher order cotangent bundles, Czech. Math. J., to appear.

Włodzimierz M. Mikulski, Institute of Mathematics, Jagiellonian University, ul. S. Lojasiewicza 6 , Kraków, Poland

e-mail: wlodzimierz.mikulski@im.uj.edu.pl 